\title{
Reconstructing the radial breathing mode resonance Raman spectra for HiPco single-wall carbon nanotubes
}

\author{
Zhengtang Luo, Rongfu Li, Sang Nyon Kim, and Fotios Papadimitrakopoulos* \\ Nanomaterials Optoelectronics Laboratory, Department of Chemistry, Polymer Program, Institute of Materials Science, \\ University of Connecticut, Storrs, Connecticut 06269-3136, USA \\ (Received 22 June 2004; published 27 December 2004)
}

\begin{abstract}
The radial breathing mode (RBM) region of the resonance Raman spectra of HiPco single-wall carbon nanotubes (SWNTs) was investigated as a function of aggregation and presence of environmental contaminants. This was modeled using an energetic deviation term $(\Delta E)$, imparted to the optical transitions $\left[E_{i i}(n, m)\right]$ by the change in SWNT physicochemical environment. Three sets of $E_{i i}(n, m)$ values were used to reconstruct these RBM profiles, based on (i) photoluminescence (PL) measurements, (ii) a simple tight-binding (TB) model, and (iii) a set of modified (TB-based) $E_{i i}(n, m)$ values to account for the underestimation of the influence of chiral angle for SWNTs with diameters below $1 \mathrm{~nm}$. The simulation revealed that the PLdetermined $E_{i i}(n, m)$ set provided a good fit in terms of peak position as opposed to TB-calculated values. Moderate improvement was attained using the third set of $E_{i i}(n, m)$ values, indicative of the importance of both curvature and chirality effects. Providing an accurate set of $E_{i i}(n, m)$ values becomes available, the RBM profile reconstruction methodology discussed herein could greatly enhance our ability to model a range of physicochemical changes to the immediate environment of SWNTs.
\end{abstract}

DOI: 10.1103/PhysRevB.70.245429

PACS number(s): 78.66.Tr, 61.48.+c

\section{INTRODUCTION}

Since the original work of Rao et al., ${ }^{1}$ where the position of the Raman band in the lower-frequency region was correlated to the radial breathing modes (RBMs) of single-wall carbon nanotubes (SWNTs), resonance Raman spectroscopy (RRS) has become the key characterization technique in this field. Visualizing SWNTs as seamless rolled-up graphite sheets, the Hamada vector $\mathbf{C}_{h}=n \mathbf{a}_{1}+m \mathbf{a}_{2}$ describes the chirality, where $n$ and $m$ are integers and $\mathbf{a}_{1}$ and $\mathbf{a}_{2}$ are the unit vectors on the graphene sheet. At present, no production method $^{2-5}$ can selectively produce a specific $(n, m)$ nanotube since the structure energy is weakly dependent on chirality. Consequently, SWNT samples appear to contain a homogeneous distribution of chiralities and diameters within a given diameter envelope that defines sample preparation. ${ }^{6}$ The large aspect ratio of SWNTs enhances the formation of bundles, in which several tubes are held together by van der Waals interactions.

RRS has been widely used to assign the $(n, m)$ for a given SWNT sample. ${ }^{7}$ A number of reports indicate that the RBM region is sensitive to the physicochemical environment ${ }^{8,9}$ and aggregation state of SWNTs. ${ }^{10,11}$ This renders the qualitative and quantitative comparison of the abundance of the specific $(n, m)$ SWNT or group of SWNTs to the RBM band of their Raman spectra problematic. Various efforts have been reported for simulating the RBM band, with moderate degree of success. ${ }^{12,6,10}$ Heller et al. ${ }^{10}$ were able to model the RBM band as a function of sample morphology using a limited set of six $(n, m)$ SWNTs. However, as will be shown in this contribution, when all possible $(n, m)$ values are included, such fitting becomes problematic.

The proper determination of optical transition energies $E_{i i}(n, m)$ of various $(n, m)$ SWNTs is important for the analysis of not only their absorption and photoluminescence spectra, ${ }^{13}$ but also for RRS. ${ }^{10}$ This is due to the fact that resonance Raman scattering can only be observed when the optical transition energy of a SWNT is close to that of the incident photon or scattered photon. ${ }^{14}$ Here, $E_{i i}(n, m)$ is the energy separation between the $i$ th pair of density of states (DOS) singularities in the valence $(v)$ and conduction $(c)$ band, where, for example, $E_{11}$ stands for the transition between $v 1$ and $c 1, E_{22}$ from $v 2$ to $c 2$, etc. Recently, a number of reports indicate substantial deviation between calculated [i.e., via tight-binding (TB) model] and experimentally determined [i.e., via scanning tunneling spectroscopy ${ }^{15,16}$ (STS) and photoluminescence $\left.{ }^{13,17}(\mathrm{PL})\right] E_{i i}(n, m)$ values. Such discrepancies might originate from various excitonic effects that appear to be especially important for smaller-diameter SWNTs. ${ }^{18}$ However, the need to address the effect of aggregation and other environmental contributors is also crucial to further improve our basic understanding of SWNTs. ${ }^{13}$

In the present study, we focused on the $785 \mathrm{~nm}$ excitation laser of HiPco SWNTs, which interrogates mostly semiconducting nanotubes, and reconstructed the RRS RBM profiles using a complete set of $E_{i i}(n, m)$ values and a single adjustable parameter $\Delta E$ that accounts for their energetic deviation caused by the change in the SWNT physicochemical environment. These were contrasted to RBM profiles collected for different sample preparation (i.e., individual and bundled SWNTs, with different degrees of environmental contaminants) and used to extract the energetic deviation $\Delta E$. This enable us to elucidate the importance of starting with a correct set of $E_{i i}(n, m)$ values and witness the shortcomings of the TB model in accounting for the proper dependence of these values with respect to curvature and chirality.

\section{EXPERIMENT}

Acid-purified HiPco SWNTs were purchased from Carbon Nanotechnologies, Inc. and used without further purifi- 
cation. The diameter distribution of the HiPco samples is believed to obey a Gaussian profile with mean diameter and variance of 0.93 and $0.2 \mathrm{~nm}$, respectively, although its outskirt reaches as far as $0.6-1.4 \mathrm{~nm} .{ }^{13,19}$ Mean diameter and variance of 1.0 and $0.2 \mathrm{~nm}$ (Ref. 20) and 1.05 and $0.15 \mathrm{~nm}$ (Ref. 6) are also reported by fitting of RBM band of HiPco SWNTs bundled samples. A few milligrams of the acid-purified $^{21}$ and deionized- (DI-)water-washed fullerene paper were annealed at $400{ }^{\circ} \mathrm{C}$ for $6 \mathrm{~h}$ in dynamic vacuum and then sealed in a glass tube under vacuum to prevent reexposure to atmospheric contaminants (i.e., $\mathrm{O}_{2}$ ). A suspension of individually isolated SWNTs was prepared using sodium dodecyl sulfate (SDS), $\mathrm{D}_{2} \mathrm{O}$, and a vacuum-annealed $\left(100{ }^{\circ} \mathrm{C}, 12 \mathrm{~h}\right)$ SWNT sample, according to O'Connell et al. ${ }^{17}$ In the present study, samples are denoted as (I) acidpurified and DI-water-washed SWNTs; (II) vacuum-annealed and sealed SWNTs; (III) $\mathrm{D}_{2} \mathrm{O}$ suspension of SDS-assisted dispersed SWNTs, which consists of mainly individually isolated SWNTs. ${ }^{17}$ Raman-scattering experiments were performed in a backscattering geometry using $785 \mathrm{~nm}$ wavelength excitation laser $\left(E_{\text {laser }}=1.579 \mathrm{eV}\right)$. Raman spectra of samples I, II, and III were taken in ambient conditions, sealed within a glass tube, and in $\mathrm{D}_{2} \mathrm{O}$ suspension, respectively.

\section{RBM RESONANCE RAMAN SPECTRA RECONSTRUCTION}

The diameter and chiral angle of a SWNT with structure $(n, m)$ can be calculated as

$$
\begin{gathered}
d(n, m)=\frac{a}{\pi} \sqrt{n^{2}+m^{2}+n m}, \\
\theta(n, m)=\tan ^{-1}[\sqrt{3} n /(n+2 m)],
\end{gathered}
$$

where $a=0.246 \mathrm{~nm}$ is the lattice constant of graphite. The resonance frequency in RBM band for SWNTs with helicity of $(n, m)[v(n, m)]$ is calculated using the following equation:

$$
v(n, m)=\frac{C_{1}}{d(n, m)}+C_{2}
$$

where $d(n, m)$ is the diameter of the SWNT with indices $(n, m)$. The parameters of $C_{1}$ and $C_{2}$ have been related to the intrinsic property of isolated SWNTs and intertube interactions, respectively. ${ }^{22}$ For isolated SWNTs, values of $C_{1}$ $=223.5 \mathrm{~cm}^{-1} \mathrm{~nm}$ and $C_{2}=12.5 \mathrm{~cm}^{-1}$ have been utilized, ${ }^{13}$ whereas for aggregated samples values of $C_{1}=239 \mathrm{~cm}^{-1} \mathrm{~nm}$ and $C_{2}=8.5 \mathrm{~cm}^{-1}$ are typically used. ${ }^{6}$ In the calculations described below, these values result in insignificant changes to the calculated line-shape and affect only the peak position of the RBM features. Within the limits of the accuracy of the calculation and considering the large diversity in literature values of $C_{1}$ and $C_{2}$, these were taken as $223.5 \mathrm{~cm}^{-1} \mathrm{~nm}$ and $12.5 \mathrm{~cm}^{-1}$, respectively. For this, the theory of Martin and Falicov ${ }^{23}$ was used and we treated the spikelike van Hove singularities as discrete energy levels. In our calculations the transition closest to the excitation laser energy is the only one considered for each $(n, m)$ SWNT species. Based on this formalism, the Raman cross section of a SWNT with structure $(n, m)[\eta(n, m)]$ was obtained from the following equation: ${ }^{23}$

$$
\begin{aligned}
\eta(n, m)= & C \\
& \times\left|\frac{M_{f} M_{\mathrm{ph}} M_{0}}{\left[\hbar \omega_{i}-E_{i i}(n, m)-i \hbar \alpha\right]\left[\hbar \omega_{s}-E_{i i}(n, m)-i \hbar \alpha\right]}\right|^{2}
\end{aligned}
$$

where $C$ is a normalization factor introduced to account for sampling conditions and the collection geometry. For this, each spectrum was normalized to its highest peak. $M_{0}$ and $M_{f}$ describe the electron-photon coupling for the ground and final states, respectively, and $M_{\mathrm{ph}}$ represents the electronphonon interaction matrix. To the first approximation, the matrix element is weakly dependent on the $E_{i i}(n, m)$ values as compared to the denominator parameters of Eq. (4), ${ }^{24}$ and therefore this product can be treated as a constant. ${ }^{22} \omega_{i}$, and $\omega_{s}$ refer to the frequencies of incident and scattered photons, respectively. $1 / \alpha$ is the lifetime of the excited electron, whose value can be calculated from the following equation:

$$
\gamma_{e}=2 \hbar \alpha,
$$

where $\gamma_{e}$ accounts for the width of singularities in the DOS, and has been deduced from STS measurements, ${ }^{15,16}$ and fitting of the $G$ band of SWNTs, ${ }^{14}$ taken to be $40 \mathrm{meV}$. Unlike Heller et al., ${ }^{10}$ who also varied the $\gamma_{e}$ value for different degrees of aggregation, in our simulations $\gamma_{e}$ was kept constant at the aforementioned value.

The RBM band of individual SWNTs is usually described by a Lorentzian line shape. ${ }^{14}$ The combined contribution to the expected RBM RRS intensity of all individual nanotubes at a specific frequency $v$ in the RBM spectra can be calculated by the following equation:

$$
I\left(v, E_{\text {laser }}\right)=\sum_{n, m} L(v, v(n, m), \Gamma) \times A(n, m) \times \eta(n, m)
$$

where $A(n, m)$ is the abundance of this type of $(n, m)$ SWNT and $\Gamma$ is the full width at half maximum (FWHM) of the Lorentzian line shape, which was assumed as $10 \mathrm{~cm}^{-1}$ for all nanotube species. ${ }^{12}$ Bundle effects and other electronic interactions of SWNTs with various chemical and environmental contaminants are likely to influence the $E_{i i}(n, m)$ values. ${ }^{25}$ The $E_{i i}(n, m)$ deviation due to bundling has been discussed previously ${ }^{10,26,27}$ and shown experimentally to yield a redshift in optical absorption with respect to individually dispersed SWNTs. ${ }^{17}$ Taking into account that the exciton binding energy $\left(\Delta E_{b}\right)$ becomes infinite for ideal one-dimensional electron-hole systems, ${ }^{25}$ the observed optical transition energy for a real SWNT sample can be rewritten as ${ }^{28}$

$$
E_{i i}^{\mathrm{obs}}(n, m)=E_{i i}(n, m)+\Delta E^{\text {Coulomb }}-\Delta E_{a}-\Delta E_{b},
$$

where $E_{i i}^{\mathrm{obs}}(n, m)$ is the apparent optical transition energy of a specific species, $\Delta E_{a}$ is the deviation from $E_{i i}(n, m)$ caused by aggregation, and $\Delta E^{\text {Coulomb }}$ is the component that increases the optical transition energy due to screened Coulomb interactions. ${ }^{28}$ Here, $\Delta E^{\text {Coulomb }}, \Delta E_{a}$, and $\Delta E_{b}$ are all 


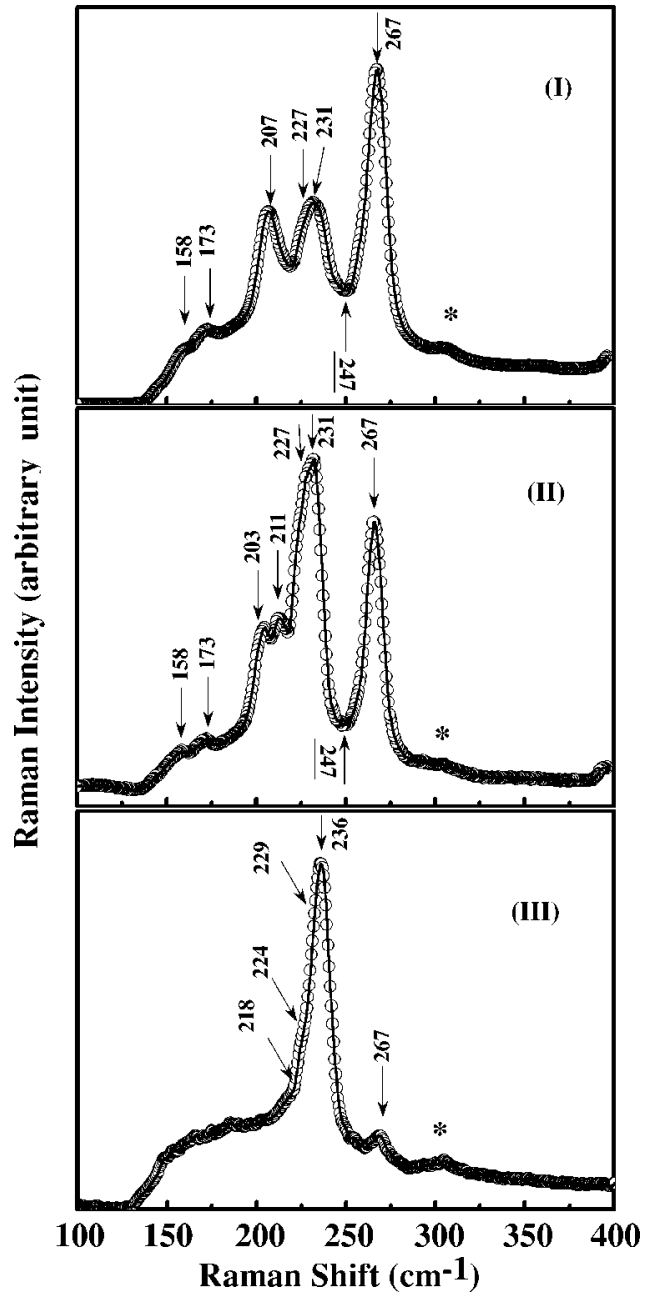

FIG. 1. RBM band of HiPco SWNTs for $E_{\text {laser }}=1.579 \mathrm{eV}$ obtained experimentally for (I) acid-purified and DI-water-washed SWNTs; (II) vacuum-annealed and sealed SWNTs; (III) $\mathrm{D}_{2} \mathrm{O}$ suspension of SDS-assisted dispersed SWNTs. The $\sim 303 \mathrm{~cm}^{-1}$ feature has a contribution from the $\mathrm{Si}$ substrate. No baseline correction was performed.

greater than 0 . As pointed out by Ichida et al., ${ }^{28}$ Coulomb effects disappear in optical transitions larger than $E_{11}$. Based on this, the energetic deviation $(\Delta E)$ of the observed optical transition of a real SWNT sample to those of theoretically calculated or experimentally measured values for isolated SWNTs and for the $E_{22}$ of semiconducting SWNTs $\left(E_{22}^{S}\right)$ can be expressed as

$$
\Delta E=E_{22}^{S}(n, m)-E_{22}^{\mathrm{obs}}(n, m)=\Delta E_{a}+\Delta E_{b} .
$$

Positive $\Delta E$ values indicate a redshift in $E_{22}^{S}$ relative to the ideal band-to-band transition without electronic perturbance.

\section{RESULTS AND DISCUSSION}

Figure 1 illustrates the RBM profiles for samples I, II, and III from the same SWNT batch when subjected to different preparation methodologies. Sample I is the as-supplied HiPco sample, which has been acid purified and extensively washed with DI water, yet exposed to air during storage. Traces of acidic functionalities plus absorbed $\mathrm{O}_{2}$ are expected to be presented in this sample. ${ }^{21}$ Sample II is obtained by annealing sample I at $400{ }^{\circ} \mathrm{C}$ for $6 \mathrm{~h}$ in dynamic vacuum (a method known ${ }^{21}$ to dissociate and clean various chemisorbed and physisorbed impurities on SWNTs) and sealed in a glass tube to further prevent $\mathrm{O}_{2}$ reexposure. Sample III is a SDS-assisted nanotube suspension that consists of mainly isolated SWNTs in $\mathrm{D}_{2} \mathrm{O} .{ }^{13}$ SWNTs can be divided into metallic (met-SWNTs) and semiconducting (sem-SWNTs) nanotubes depending on whether or not $|n-m|$ is an integer divisible by $3 .{ }^{14}$ For HiPco samples, the RBM spectra obtained from $785 \mathrm{~nm}$ wavelength laser excitation (shown in Fig. 1) are mainly associated with $E_{22}^{S}$ transition energies of sem-SWNTs. ${ }^{29}$ For samples I and II, the arrow-indicated Raman features match closely in peak positions, yet differ significantly in their relative peak intensities. Moreover, while samples I and II exhibit multiple peaks between 150 and $300 \mathrm{~cm}^{-1}$, sample III shows mainly an asymmetry peak centered at ca. $236 \mathrm{~cm}^{-1}$. Composition variation can be easily ruled out as the origin of such a line shape change since a spectrum similar to sample II has been obtained if the $\mathrm{D}_{2} \mathrm{O}$ in sample III is evaporated (data not shown). Furthermore, with the exception of zigzag nanotubes, the HiPco preparation route favors no individual chiralities and the SDS-assisted dispersion are less likely to cause solubility differences among different diameter and chirality nanotubes. ${ }^{13}$ The variance between samples I and II appears to be concentrated mainly at the relative peak height of the 231 and $267 \mathrm{~cm}^{-1}$ features, with both samples showing a big valley (at $247 \mathrm{~cm}^{-1}$ ) between these two peaks. The RBM bands for samples I and II are entirely reproducible on different spots of the 25 spots collected for each sample, bearing witness to sample homogeneity. However, the spectrum collected for sample III changes slightly depending on the different spots or different collection times at the same spot. These observations signify that considerable attention to sample preparation must be exerted before one makes conclusions about composition variation using RBM line shape analysis.

Similar RBM spectral variation was observed by Rao et al. ${ }^{27}$ for $\mathrm{CS}_{2}$-solubilized SWNT as opposed to bundled samples. These researchers have described such differences as upshifts of various RBM peaks when SWNTs are in a debundled state. This, however, is in contradiction to the $6-20 \mathrm{~cm}^{-1} \mathrm{RBM}$ band downshift predicted by theoretical analysis. ${ }^{14,30}$ Such contradiction is explained as involving narrowing of the optical transition energy in isolated tubes over that in ropes. ${ }^{27}$ Recent experimental observations, however, suggest that a redshift in optical transition is more likely for bundled SWNTs, ${ }^{10,17}$ casting doubts on the aforementioned $E_{i i}(n, m)$ narrowing explanation.

In order to understand the influence of sample preparation on their resonant Raman spectra, the RBM band was reconstructed using the aforementioned simulation methods. The strong coupling of the Raman cross section to the optical transition energies $E_{i i}(n, m)$ as well as the energetic deviation [ $\Delta E$, defined as $\left.E_{i i}(n, m)-E_{i i}^{\mathrm{obs}}(n, m)\right]$ is the key in determinating the resonance conditions. Here we assume that sample bundling and air/acid exposure history will affect the optical transition detected (mainly the second optical transition of 


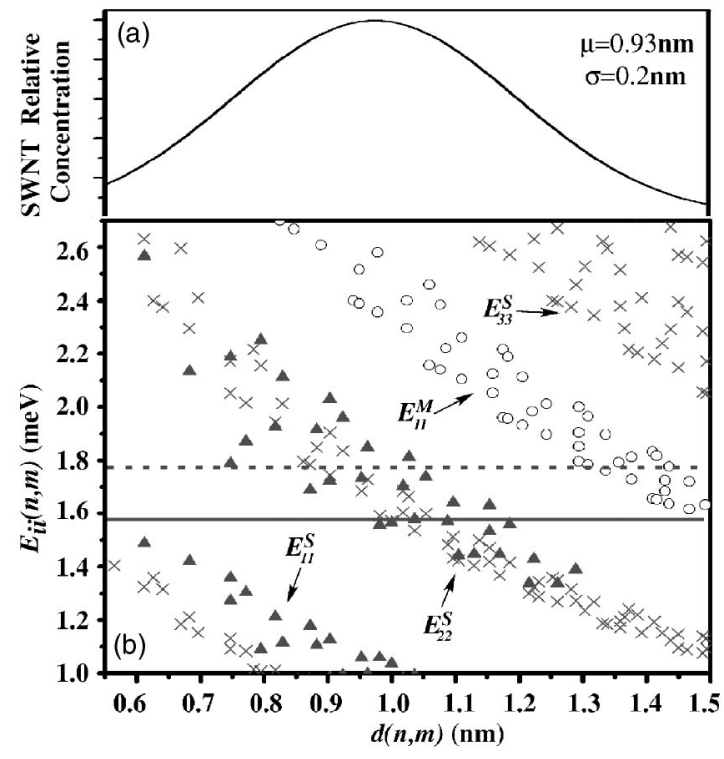

FIG. 2. (a) Concentration distribution of HiPco SWNTs, and (b) optical transition energies $E_{i i}(n, m)$ as a function of nanotube diameter (Kataura plot). Circles and crosses are for met-SWNTs and sem-SWNTs, respectively, obtained from simple tight-binding model (Refs. 31-33) Solid trigonals depict $E_{i i}(n, m)$ observed by photoluminescence measurements (Ref. 13). The horizontal solid line indicates the excitation laser energy and the dotted line represents the imaginary laser energy equivalent to a downshift of $E_{i i}(n, m)$ by $200 \mathrm{meV}$.

semiconducting SWNTs, $E_{22}^{S}$ ) and their relative contribution remains to be determined. For both effects, the $\Delta E$ is taken to be $(n, m)$ independent. This assumption needs further assessment and will be the subject of future studies. Applying such $\Delta E$ to the $E_{i i}(n, m)$ is equivalent to an upshift of the $E_{\text {laser }}$ by the same amount. This is schematically illustrated in Fig. 2(b), which makes it easier to visualize the changes in resonance conditions for SWNTs with different $(n, m)$ values. In this study, the $E_{i i}(n, m)$ are obtained from experimental results from photoluminescence of sem-SWNTs ${ }^{13}$ and theoretical results following the tight binding of graphene using the zone-folding procedure taking the nearest neighbor carboncarbon overlapping integral $\gamma_{0}$ to be $2.9 \mathrm{eV} \cdot{ }^{31-33}$ Figure 2(a) illustrates the Guassian distribution of HiPco SWNT concentration as a function of diameter ${ }^{13,19}$ along with the Kataura plot $^{31}$ [Fig. 2(b)] of the $E_{i i}(n, m)$ values obtained by the aforementioned sources. The $785 \mathrm{~nm}(1.579 \mathrm{eV})$ excitation laser is primarily in resonance with $E_{22}^{S}$ although a small fraction of small-diameter sem-SWNTs with $E_{11}^{S}$ and largerdiameter met-SWNTs with $E_{11}^{M}$ could also be in weak resonance as well, shown on either ends of Fig. 2(b).

Figure 3 demonstrates the Raman cross section, obtained from Eq. (4) as a function of TB-calculated $E_{22}^{S}(n, m)$ values. As mentioned above, $\Delta E$ accounts for the redshift induced in $E_{22}^{S}(n, m)$ that can originate from variation of the immediate environment of the SWNTs (i.e., aggregation, doping, etc.). A $\Delta E$ of $200 \mathrm{meV}$ (depicted in both Figs. 2 and 3) represents an upper limit for its value and was chosen to readily visualize the profound shift in resonant conditions. In reality, much smaller values (on the order of ca. $100 \mathrm{meV}$ ) have

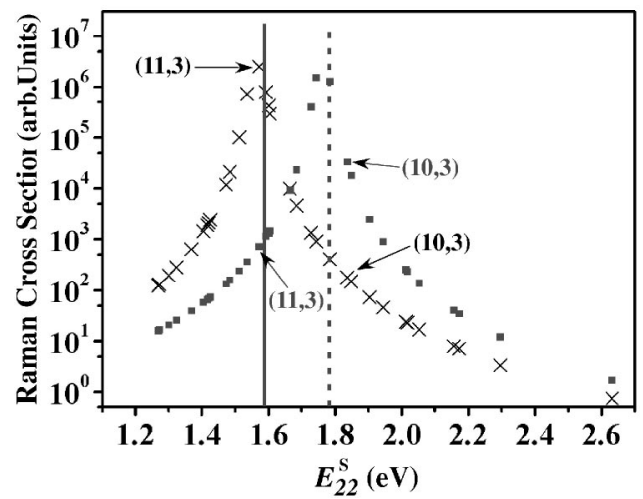

FIG. 3. Calculated Raman cross section as a function of TBdetermined $E_{22}^{S}(n, m)$, for 0 (crosses) and 200 (rectangles) meV $\Delta E$ values under $1.579 \mathrm{eV}$ laser excitation. Solid and dotted vertical lines refer to the notation used in Fig. 2.

been utilized in the literature ${ }^{10,17,34}$ as well as this study. For example, in case of $\Delta E=0 \mathrm{meV}$ for $E_{22}(11,3)=1.572 \mathrm{eV}$ and $E_{22}(10,3)=1.836 \mathrm{eV}$, the Raman cross section ratio $\eta(11,3) / \eta(10,3)=14000$, while their abundance ratio $A(11,3) / A(10,3)$ is only 0.94 . Taking into consideration the logarithmic scale of the Raman cross section in Fig. 3, it is clear that a slight change of $\Delta E$ value will lead to considerable difference in the Raman RBM band. For example, for a $(11,3)$ SWNT the resonance factor ratio becomes 3400:1 for $\Delta E$ values of 0 and $200 \mathrm{meV}$, respectively.

Figure 4 illustrates how each SWNT is defined by a pair of integer $(n, m)$ values. The SWNT diameter $d(n, m)$ and corresponding Raman shift are also depicted using Eqs. (1) and (3) to provide a guide for RBM RRS interpretation. With the exception of the $(10,10)$ and $(11,11)$ armchair metSWNTs, all other met-SWNTs are omitted because they cannot be observed in PL measurement and most of them are in weak resonance with the laser used. $(10,10)$ and $(11,11)$ are included in some of the simulations using tight-binding calculated $E_{11}^{M}(n, m)$ values, since they are close to $E_{\text {laser, }}$ and may be favored during the HiPco growth process. ${ }^{13}$ Moreover, when $\Delta E$ is applied, their $E_{11}^{M}(n, m)$ values will get closer to $E_{\text {laser, }}$ as shown in Fig. 2. The individual SWNTs corresponding to gray-coded hexagons have been observed in PL experiments. ${ }^{13}$ The sem-SWNTs denoted with deep gray are primarily in resonance with the $785 \mathrm{~nm}$ laser, as opposed to those with light gray. As will become clear later in the calculation, keeping the light gray species or not in the calculation makes no difference to the RBM profile. The hatched area represents the region in which the TBdetermined $E_{i i}(n, m)$ values contain considerable error and require further reevaluation. ${ }^{35,36}$

Figures 5(a)-5(i) illustrate the simulated RBM profiles and corresponding $\Delta E$ values. Herein, the $\Delta E$ was adjusted to obtain the best possible fit while maintaining a comparable line shape between the simulated and experimental data. Figures 5(a)-5(c) were attained using the $E_{i i}(n, m)$ values obtained from PL measurements for samples III, II, and I, respectively. The simulated spectra are in good agreement in predicting the peak positions of the experimentally obtained spectra. For the isolated nanotube of Fig. 1 (III) [also shown 


\section{Raman Shift $\left(\mathrm{cm}^{-1}\right)$}

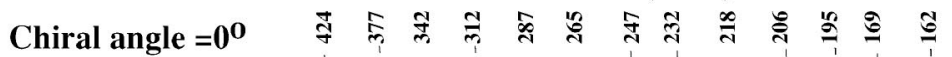

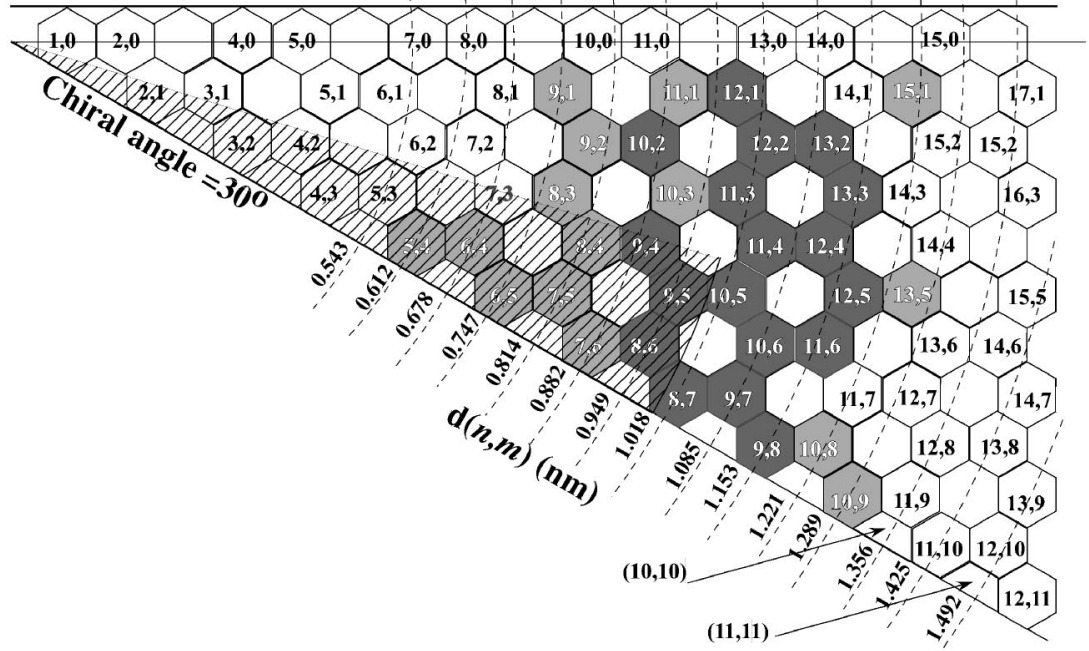

FIG. 4. Chirality map for HiPco SWNT. Shaded cells indicate the sem-SWNTs observed by photoluminescence measurements. Deep and light shaded cells represent strong and weak resonance, respectively, under $785 \mathrm{~nm}$ $(1.579 \mathrm{eV})$ laser excitation. The hatched triangular section depicts the sem-SWNTs with tightbinding model calculated $E_{i i}(n, m)$ values having considerable error, due to small diameter and high chiral angle. in Fig. 5(a)], the satellite features $\left(218,224\right.$, and $\left.229 \mathrm{~cm}^{-1}\right)$ to the $236 \mathrm{~cm}^{-1}$ main peak have been predicted with either peaks or shoulders in the simulated spectrum of Fig. 5(a). The fact that there is no need to employ a value of $\Delta E$ (i.e., $\Delta E=0)$ signifies the close correlation of Raman spectroscopy with the optical and PL measurements, as well as the viability of this method. The appearance of a single main peak at ca. $237 \mathrm{~cm}^{-1}$ originates from the fact that the differences in $v(n, m)$ values are smaller than their FWHMs, and thus sev- eral peaks are grouped together as one. This implies that assigning a RBM Raman peak to a single $(n, m)$ value is questionable, let alone simulating the entire RBM region with a selected limited set of SWNTs. ${ }^{10}$ Moreover, as will become clear later on in the discussion, the small peak at $\sim 267 \mathrm{~cm}^{-1}$ is evidence of the existence of a small amount of bundles in the suspension of individual SWNTs. ${ }^{35}$

Pronounced RBM line shape changes for samples II and I can be realized if one increases the $\Delta E$ value. In terms of

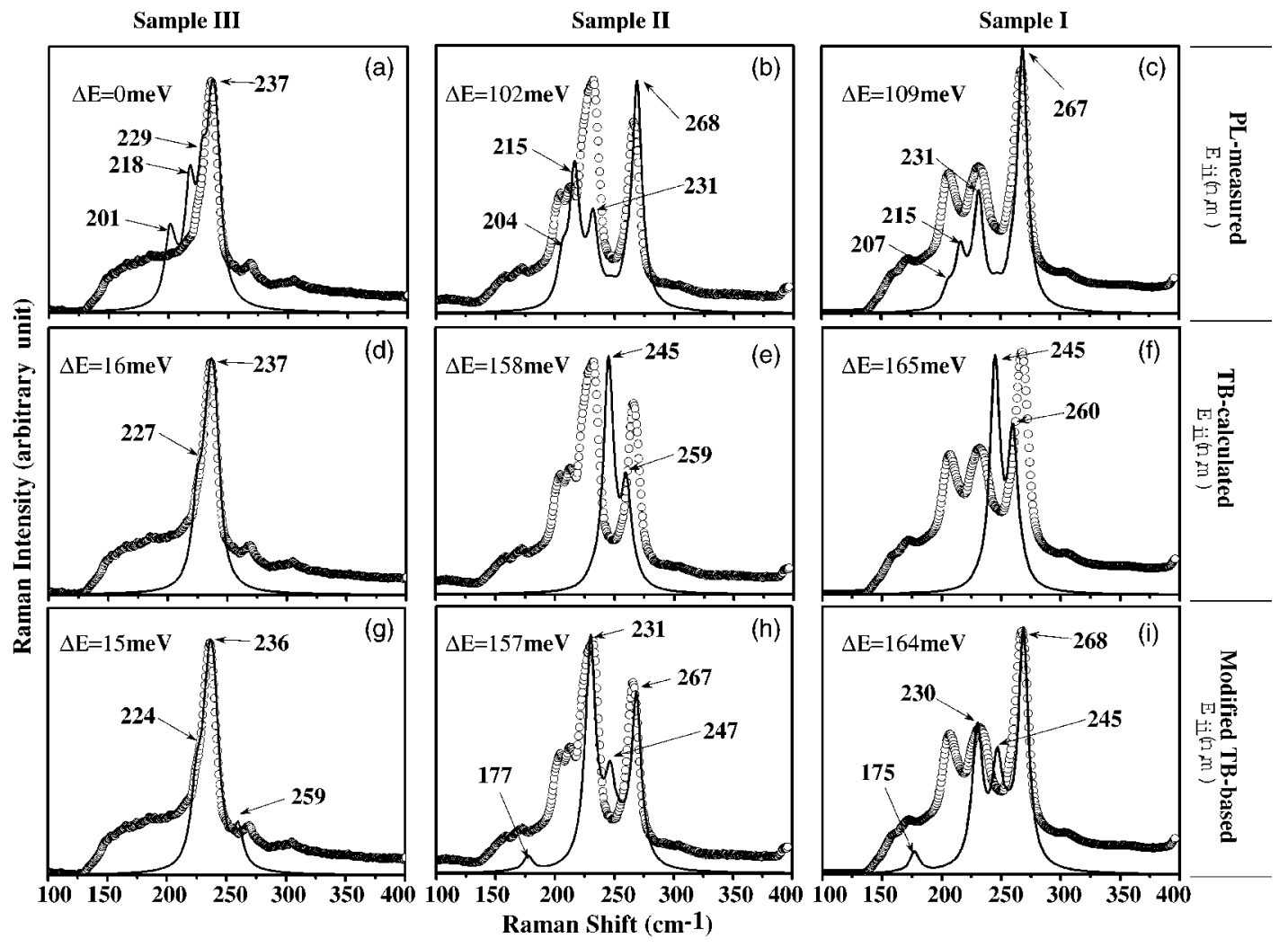

FIG. 5. Experimental (open circles) and simulated (solid lines) RBM spectra arranged vertically for samples III, II, and I and horizontally for various sets of $E_{i i}(n, m)$ values (see text for details). Marked peaks refer to simulated spectra. 
peak position and peak ratio, the best fit to the RBM band of samples II and I has been achieved at $\Delta E$ of 102 and $109 \mathrm{meV}$, respectively [see Figs. 5(b) and 5(c)]. One of the remarkable attributes of the PL-determined $E_{i i}(n, m)$ values is that they accurately predict the $247 \mathrm{~cm}^{-1}$ valley between the sharp 231 and $267 \mathrm{~cm}^{-1}$ peaks. Moreover, the experimentally observed features at ca. 203, 211, 227, 231, and $267 \mathrm{~cm}^{-1}$ are close to the simulated 204, 215, 231, and $267 \mathrm{~cm}^{-1}$ features for sample II. Similarly, the experimentally observed 207, 231, and $267 \mathrm{~cm}^{-1}$ features of sample I come equally close to the simulated 207, 215, 231, and $267 \mathrm{~cm}^{-1}$ features. The downshift of $E_{i i}(n, m)$ values, or imaginary upshift of $E_{\text {laser }}$, brings also into resonance the larger-diameter metallic SWNTs, as evidenced by the 158 and $173 \mathrm{~cm}^{-1}$ features for the bundled samples II and I. Assuming there is no change to any excitonic effects before and after bundling (i.e., $\Delta E_{b}=0$ ), a value of $102 \mathrm{meV}$ for $\Delta E_{a}$ can be obtained from Fig. 5(b) according to Eq. (8).

The electronic properties of carbon nanotubes are know to be influenced by various environmental contaminants as shown by scanning tunneling spectroscopy ${ }^{34}$ and nuclear magnetic resonance experiments, ${ }^{37}$ although the mechanism is still arguable. ${ }^{38}$ The band gap change of sem-SWNTs after exposure to air has been reported to be as high as $150 \mathrm{meV}$, and causes them to even behave as met-SWNTs. ${ }^{34}$ In Fig. 5 (c), the $\Delta E$ value of $109 \mathrm{meV}$ can be taken as the sum of $\Delta E_{a}$ and $\Delta E_{b}$ according Eq. (8). Assuming that the aggregation state is the same between samples II and I, then a value of $\Delta E_{b}=7 \mathrm{meV}$ can be obtained that could be attributed to the excitonic effect induced by air/trace acid exposure history. However, this is far from the actual picture since upon removal of these contaminants, significant changes might occur with respect to the localized nanotube environments, ${ }^{39-41}$ and will be the topic of investigations in subsequent studies.

The large variation in peak intensities between the simulated and experimental spectra could be readily explained based on the fact that an average error of $25 \mathrm{meV}$ is typically associated with PL measurements. Such fluctuations of $E_{i i}(n, m)$ values are expected to considerably alter their Raman cross sections (shown in Fig. 3) and thus peak intensities. Increasing the $\gamma_{e}$ and/or $\Gamma$ value, the FWHM of the Lorentzian line shape was found to broaden the simulated features, although it resulted in minimum changes in peak intensities. Although SWNT aggregation and other changes in their immediate environment are expected to influence $\gamma_{e}$ and/or $\Gamma$, further research is needed to incorporate it in a rational fashion. As an example, Fig. S1 (Ref. 42) illustrates a simulation comparison when the linewidth is changed from 10 to 8 and $6 \mathrm{~cm}^{-1}$. The peaks become sharper when $\Gamma$ is decreased, although the peak positions do not get affected.

As previously discussed, the line shape of the RBM spectrum is mostly affected by the $E_{i i}(n, m)$ values and less by the SWNT natural abundance. Figure S2 (Ref. 42) illustrates a comparison using the aforementioned three reported diameter distributions for HiPco SWNTs. Here, although the relative RBM lineshape is retained, small relative peak intensity variations are witnessed.

The theoretically derived $E_{i i}(n, m)$ values from the tightbinding model were also subjected to a similar fitting process for reconstructing the RBM band of these three samples. Figures 5(d)-5(f) show the fits corresponding to samples III, II, and I, respectively. As mentioned above, $E_{11}^{M}$ of $(10,10)$ and $(11,11)$ are also included, since they are closer in resonance with $E_{\text {laser }}$, and evident from the discernible 158 and $173 \mathrm{~cm}^{-1}$ features. The best fit to the experimental data of sample III was obtained with a $\Delta E$ value of $16 \mathrm{meV}$. Such $16 \mathrm{meV}$ values might arise from the micelle SDS enclosure and/or various inaccuracies of the TB model. The overlap integral $\gamma_{0}$, which typically varies from 2.5 to 3 , in this study was fixed at a value of 2.9. Shifting the $\gamma_{0}$ to 3.0 will shift the $\Delta E$ value from 16 to $55 \mathrm{meV}$ (data not shown).

In the case of the aggregated samples (i.e., II and I), Figures 5(e) and 5(f) illustrate a very poor fit to the experimental data, even if $\Delta E$ ranged from 0 to $400 \mathrm{meV}$. In particular, the valley at $247 \mathrm{~cm}^{-1}$ is impossible to reproduce using this set of $E_{i i}(n, m)$ values. For SWNTs that have diameters smaller than $1 \mathrm{~nm}$ and chiral angles close to $30^{\circ}$ (see hatched area in Fig. 4), the trigonal warping effect, ${ }^{32}$ which is proportional to $[\theta(n, m) / d(n, m)]^{2}$ along with curvature and overlap effects, ${ }^{32}$ becomes very important to the electronic properties of these SWNTs. As shown in the Kataura plot of Fig. 2(b), the PL-determined $E_{i i}(n, m)$ values of such SWNTs deviate considerably from the values predicted by the TB model. Such deviations vary greatly with the $(n, m)$ indices and it appears that the relative placements do not obey a certain trend that can be fitted adequately by changing the $\Delta E$ value. One of the reasons might be that the excitonic binding energy is dependent on both diameter and chiral angle. $^{25,28,43}$

Modification of the TB-obtained $E_{i i}(n, m)$ values is therefore needed in order to improve fitting. Lacking a firm theory to calculate these values explicitly, we were forced to tentatively constructed a modified Kataura plot by shifting the $E_{i i}(n, m)$ values of the hatched area in Fig. 4 by the following equation: ${ }^{13}$

$$
\Delta E_{i i}(n, m)=A \times \frac{\cos [3 \theta(n, m)]}{[d(n, m)]^{2}},
$$

where $A$ is a constant depending on the chirality and the associated optical transition. In particular, the empirically deduced values for $A$ of -0.0883 and $0.0459 \mathrm{~nm}^{2} \mathrm{eV}$ were used for $E_{11}$ when $(n-m) \bmod 3=1$ and 2, respectively. ${ }^{13}$ Similarly, in the case of $E_{22}$, values of 0.1709 and $-0.1834 \mathrm{~nm}^{2} \mathrm{eV}$ were used when $(n-m) \bmod 3=1$ and 2, respectively. Figures 5(g)-5(i) show the corresponding RBM fits using such modified Kataura plot. For samples II and I, the $247 \mathrm{~cm}^{-1}$ valley reemerges. Interestingly, although the changes performed to the Kataura plot were far away from the $(10,10)$ and $(11,11)$ met-SWNTs, the low-wave-number features (ca. 158, $173 \mathrm{~cm}^{-1}$ ) features are now prominent in Figs. 5(h) and 5(i) as opposed to Figs. 5(e) and 5(f). This is representative of the relative intensity distribution among various nanotubes. Thus, if the $E_{i i}(n, m)$ dependence on chirality is small, fewer nanotubes are in resonance, monopolizing the majority of the RBM intensity, which essentially hides the others. Spreading the $E_{i i}(n, m)$ values over a larger range distributes the intensity to more SWNT species 
and thereby provides the opportunity for species that were otherwise buried in the background to stand out. In terms of $\Delta E$, values of 15,157 , and $164 \mathrm{meV}$ were employed to obtain the best fits for samples III, II, and I, respectively. Using a similar formalism discussed before, values of 147 and $7 \mathrm{meV}$ for $\Delta E_{a}$ and $\Delta E_{b}$ were derived. The existence of a small peak at $247 \mathrm{~cm}^{-1}$, however, mainly contributed from the $(8,6)$ SWNT, indicates that such an empirical modification to the $E_{i i}(n, m)$ values is far from perfect. If this chirality modification, performed for the hatched area, is applied to all shaded nanotubes, the fitting becomes once again quite poor (data not shown). The strong chiral dependence of $E_{i i}(n, m)$ values is believed to be particularly important for smalldiameter SWNTs. ${ }^{13,36}$ Kurti et al. ${ }^{44}$ have argued that considerable hexagonal distortions can be expected for smallerdiameter SWNTs of high chiral angle, which in turn affect greatly their $E_{i i}(n, m)$ values. The TB model also failed to accurately predict the observed RBM spectrum at the lowwave-number region (i.e., $205-225 \mathrm{~cm}^{-1}$ ). A closer investigation of the Kautara plot in Fig. 2 indicates that the TB model underestimated the $E_{i i}(n, m)$ values of larger-diameter SWNTs compared to the PL-determined values. This places the $E_{i i}(n, m)$ values farther from the laser energy, a situation that becomes worse when a positive $\Delta E$ is included. More work is needed to understand this phenomenon.

\section{CONCLUSION}

The RBM region of the resonance Raman spectrum of SWNT was found to be significantly influenced by the physicochemical environment in the vicinity of these SWNTs and their aggregation state. Reconstruction of these RBM profiles using the entire set of $(n, m)$ nanotubes provided a more quantitative assessment of the deviations of $E_{i i}(n, m)$ values from those in their isolated states, which was attributed to bundling and possibly excitonic effects. Such spectral reconstruction sheds more light on the inability of the simple tightbinding model to accurately predict the RBM band line shape because it underestimates the importance of the chiral angle in determination of the $E_{i i}(n, m)$ values of smalldiameter SWNTs. Assuming the correct set of $E_{i i}(n, m)$ values is available, such RBM band reconstruction methodology could greatly enhance our ability to model a range of physicochemical changes to the immediate environment of SWNTs.

\section{ACKNOWLEDGMENTS}

The authors would like to thank Professor Ado Jorio de Vasconcelos and G. Lavigne for helpful discussions. Financial support from AFOSR Grants No. F49620-01-1-0545 and No. ARODAAD-19-02-1-0381 is greatly appreciated.
*Author to whom correspondence should be addressed. Email address: papadim@mail.ims.uconn.edu

${ }^{1}$ A. M. Rao, E. Richter, S. Bandow, B. Chase, P. C. Eklund, K. A. Williams, S. Fang, K. R. Subbaswamy, M. Menon, A. Thess, R. E. Smalley, G. Dresselhaus, and M. S. Dresselhaus, Science 275, 187 (1997).

${ }^{2}$ Y. Saito, T. Yoshikawa, S. Bandow, M. Tomita, and T. Hayashi, Phys. Rev. B 48, 1907 (1993).

${ }^{3}$ A. Thess et al., Science 273, 483 (1996).

${ }^{4}$ J. H. Hafner, C.-L. Cheung, T. H. Oosterkamp, and C. M. Lieber, J. Phys. Chem. B 105, 743 (2001).

${ }^{5}$ M. J. Bronikowski, P. A. Willis, D. T. Colbert, K. A. Smith, and R. E. Smalley, J. Vac. Sci. Technol. A 19, 1800 (2001).

${ }^{6}$ A. Kukovecz, C. Kramberger, V. Georgakilas, M. Prato, and H. Kuzmany, Eur. Phys. J. B 28, 223 (2002).

${ }^{7}$ A. Jorio, R. Saito, J. H. Hafner, C. M. Lieber, M. Hunter, T. McClure, G. Dresselhaus, and M. S. Dresselhaus, Phys. Rev. Lett. 86, 1118 (2001).

${ }^{8}$ P. H. Tan, C. Y. Hu, F. Li, S. Bai, P. X. Hou, and H. M. Cheng, Carbon 40, 1131 (2002).

${ }^{9}$ A. Kukovecz, T. Pichler, R. Pfeiffer, C. Kramberger, and H. Kuzmany, Phys. Chem. Chem. Phys. 5, 582 (2003).

${ }^{10}$ D. A. Heller, P. W. Barone, J. P. Swanson, R. M. Mayrhofer, and M. S. Strano, J. Phys. Chem. B 108, 6905 (2004).

${ }^{11}$ H. Kataura, Y. Maniwa, S. Masubuchi, S. Kazama, X. Zhao, Y. Ando, Y. Ohtsuka, S. Suzuki, Y. Achiba, and R. Saito, in Electronic Properties of Novel Material-Molecular Nanostructures, edited by H. Kuzmany, J. Fink, M. Mehring, and S. Roth, AIP Conf. Proc. No. S44 (AIP, Melville, NY, 2000), p. 262.
${ }^{12}$ E. Richter and K. R. Subbaswamy, Phys. Rev. Lett. 79, 2738 (1997).

${ }^{13}$ S. M. Bachilo, M. S. Strano, C. Kittrell, R. H. Hauge, R. E. Smalley, and R. B. Weisman, Science 298, 2361 (2002).

${ }^{14}$ M. S. Dresselhaus and P. C. Eklund, Adv. Phys. 49, 705 (2000).

${ }^{15}$ J. W. G. Wildoer, L. C. Venema, A. G. Rinzier, R. E. Smalley, and C. Dekker, Nature (London) 391, 59 (1998).

${ }^{16}$ T. W. Odom, J.-L. Huang, P. Kim, and C. M. Lieber, Nature (London) 391, 62 (1998).

${ }^{17}$ M. J. O’Connell, S. M. Bachilo, C. B. Huffman, V. C. Moore, M. S. Strano, E. H. Haroz, K. L. Rialon, P. J. Boul, W. H. Noon, C. Kittrell, J. Ma, R. H. Hauge, R. B. Weisman, and R. E. Smalley, Science 297, 593 (2002).

${ }^{18}$ C. D. Spataru, S. Ismail-Beigi, L. X. Benedict, and S. G. Louie, Phys. Rev. Lett. 92, 077402 (2004).

${ }^{19}$ M. Zheng, A. Jagota, M. S. Strano, A. P. Santos, P. Barone, S. G. Chou, B. A. Diner, M. S. Dresselhaus, R. S. McLean, G. B. Onoa, G. G. Samsonidze, E. D. Semke, M. Usrey, and D. J. Walls, Science 302, 1545 (2003).

${ }^{20}$ G. G. Samsonidze, S. G. Chou, A. P. Santos, V. W. Brar, G. Dresselhaus, M. S. Dresselhaus, A. Selbst, A. K. Swan, M. S. Unlu, B. B. Goldberg, D. Chattopadhyay, S. N. Kim, and F. Papadimitrakopoulos, Appl. Phys. Lett. 85, 1006 (2004).

${ }^{21}$ I. W. Chiang, B. E. Brinson, A. Y. Huang, P. A. Willis, M. J. Bronikowski, J. L. Margrave, R. E. Smalley, and R. H. Hauge, J. Phys. Chem. B 105, 8297 (2001).

${ }^{22}$ H. Kuzmany, W. Plank, M. Hulman, C. Kramberger, A. Gruneis, T. Pichler, H. Peterlik, H. Kataura, and Y. Achiba, Eur. Phys. J. B 22, 307 (2001). 
${ }^{23}$ R. M. Martin and L. M. Falicov, Top. Appl. Phys. 8, 79 (1975).

${ }^{24}$ A. G. Souza Filho, A. Jorio, J. H. Hafner, C. M. Lieber, R. Saito, M. A. Pimenta, G. Dresselhaus, and M. S. Dresselhaus, Phys. Rev. B 63, 241404 (2001).

${ }^{25}$ T. Ando, J. Phys. Soc. Jpn. 66, 1066 (1997).

${ }^{26}$ A. M. Rao, P. C. Eklund, S. Bandow, A. Thess, and R. E. Smalley, Nature (London) 388, 257 (1997).

${ }^{27}$ A. M. Rao, J. Chen, E. Richter, U. Schlecht, P. C. Eklund, R. C. Haddon, U. D. Venkateswaran, Y. K. Kwon, and D. Tomanek, Phys. Rev. Lett. 86, 3895 (2001).

${ }^{28}$ M. Ichida, S. Mizuno, Y. Saito, H. Kataura, Y. Achiba, and A. Nakamura, Phys. Rev. B 65, 241407 (2002).

${ }^{29}$ M. Zheng, A. Jagota, E. D. Semke, B. A. Diner, R. S. McLean, S. R. Lustig, R. E. Richardson, and N. G. Tassi, Nat. Mater. 2, 338 (2003).

${ }^{30}$ U. D. Venkateswaran, A. M. Rao, E. Richter, M. Menon, A. Rinzler, R. E. Smalley, and P. C. Eklund, Phys. Rev. B 59, 10928 (1999).

${ }^{31}$ H. Kataura, Y. Kumazawa, Y. Maniwa, I. Umezu, S. Suzuki, Y. Ohtsuka, and Y. Achiba, Synth. Met. 103, 2555 (1999).

${ }^{32}$ R. Saito, G. Dresselhaus, and M. S. Dresselhaus, Phys. Rev. B 61, 2981 (2000).

${ }^{33}$ S. Maruyama, http://www.photon.t.u-tokyo.ac.ip/ maruyama/

${ }^{34}$ P. G. Collins, K. Bradley, M. Ishigami, and A. Zettl, Science 287, 1801 (2000).

${ }^{35}$ M. S. Strano, C. A. Dyke, M. L. Usrey, P. W. Barone, M. J. Allen,
H. Shan, C. Kittrell, R. H. Hauge, J. M. Tour, and R. E. Smalley, Science 301, 1519 (2003).

${ }^{36}$ A. G. Souza Filho, S. G. Chou, G. G. Samsonidze, G. Dresselhaus, M. S. Dresselhaus, L. An, J. Liu, A. K. Swan, M. S. Unlu, B. B. Goldberg, A. Jorio, A. Gruneis, and R. Saito, Phys. Rev. B 69, 115428 (2004).

${ }^{37}$ X.-P. Tang, A. Kleinhammes, H. Shimoda, L. Fleming, K. Y. Bennoune, S. Sinha, C. Bower, O. Zhou, and Y. Wu, Science 288, 492 (2000).

${ }^{38}$ A. Goldoni, R. Larciprete, L. Petaccia, and S. Lizzit, J. Am. Chem. Soc. 125, 11329 (2003).

${ }^{39}$ J. Zhao, H. Park, J. Han, and J. P. Lu, J. Phys. Chem. B 108, 4227 (2004).

${ }^{40}$ J. Zhao, J. Han, and J. P. Lu, Phys. Rev. B 65, 193401 (2002).

${ }^{41}$ K.-i. Okazaki, Y. Nakato, and K. Murakoshi, Phys. Rev. B 68, 035434 (2003).

${ }^{42}$ See EPAPS Document No. E-PRBMDO-70-061448 for two supplementary figures and more detailed explanation. A direct link to this document may be found in the online article's HTML reference section. The document may also be reached via the EPAPS homepage (http://www.aip.org/pubservs/epaps.html) or from ftp.aip.org in the directory /epaps/. See the EPAPS homepage for more information.

${ }^{43}$ T. G. Pedersen, Carbon 42, 1007 (2004).

${ }^{44}$ J. Kurti, V. Zolyomi, M. Kertesz, and G. Sun, New J. Phys. 5, 125 (2003). 